\title{
A present-day aminoacyl-tRNA synthetase with ancestral editing properties
}

\author{
BIN ZHU, ${ }^{1}$ MING-WEI ZHAO, ${ }^{1}$ GILBERT ERIANI, ${ }^{2}$ and EN-DUO WANG ${ }^{1}$ \\ ${ }^{1}$ State Key Laboratory of Molecular Biology, Institute of Biochemistry and Cell Biology, Shanghai Institutes for Biological Sciences, The Chinese \\ Academy of Sciences, and Graduate School of the Chinese Academy of Sciences, Shanghai 200031, People's Republic of China \\ ${ }^{2}$ UPR9002 du CNRS, ARN, Université Louis Pasteur, 67084 Strasbourg, France
}

\begin{abstract}
Leucyl-, isoleucyl-, and valyl-tRNA synthetases form a subgroup of related aminoacyl-tRNA synthetases that attach similar amino acids to their cognate tRNAs. To prevent amino acid misincorporation during translation, these enzymes also hydrolyze mischarged tRNAs through a post-transfer editing mechanism. Here we show that LeuRS from the deep-branching bacterium Aquifex aeolicus edits the complete set of aminoacylated tRNAs generated by the three enzymes: Ile-tRNA ${ }^{\text {Ile }}$, Val-tRNA ${ }^{\text {Ile }}$, ValtRNA $^{\text {Val }}$, Thr-tRNA ${ }^{\mathrm{Val}}$, and Ile-tRNA ${ }^{\text {Leu }}$. This unusual enlarged editing property was studied in a model of a primitive editing system containing a composite minihelix carrying the triple leucine, isoleucine, and valine identity mimicking the primitive tRNA precursor. We found that the freestanding LeuRS editing domain can edit this precursor in contrast to IleRS and ValRS editing domains. These results suggest that $A$. aeolicus LeuRS carries editing properties that seem more primitive than those of IleRS and ValRS. They suggest that the $A$. aeolicus editing domain has preserved the ambiguous editing property from the ancestral common editing domain or, alternatively, that this plasticity results from a specific metabolic adaptation.
\end{abstract}

Keywords: Aminoacyl-tRNA synthetases; evolution; proofreading

\section{INTRODUCTION}

Aminoacyl-tRNA synthetases (aaRS) link amino acids to their cognate tRNAs in aminoacylation reactions that establish the connection between a specific amino acid and the anticodon triplet of the tRNA (Schimmel 1987; Ibba and Söll 2000). AaRS are divided into two classes of 10 enzymes each, based on the active-site architecture shared by all members of the same class (Eriani et al. 1990; Cusack et al. 2000). Class I- and II-defining domains are the ancient, historical aaRS that appeared at the very beginning of the protein world. Later in evolution, peptide insertions were added to these central domains, and additional $\mathrm{N}$ - or C-terminal domains were progressively appended. One crucial function provided by domain insertions is the editing of mischarged tRNAs. Editing domains consist of a second active site designed to hydrolytically clear errors of aminoacylation. Editing activities have been characterized in class Ia aaRS, including IleRS, ValRS, and LeuRS (Eldred and Schimmel

Reprint requests to: E.-D. Wang, 320 Yue Yang Road, Shanghai, China 200031; e-mail: edwang@sibs.ac.cn; fax: 86-21-54921011.

Article published online ahead of print. Article and publication date are at http://www.rnajournal.org/cgi/doi/10.1261/rna.228707.
1972; Fersht and Kaethner 1976; Chen et al. 2000), as well as class II aaRS, including ThrRS, ProRS, AlaRS, and PheRS (Beuning and Musier-Forsyth 2000; Dock-Bregeon et al. 2000; Beebe et al. 2003; Roy et al. 2005). Four designs for these editing sites have been described, one for class I enzymes (Nureki et al. 1998) and three for class II enzymes (Dock-Bregeon et al. 2000; Wong et al. 2003; Kotik-Kogan et al. 2005; Ishijima et al. 2006). The class I editing site of IleRS, ValRS, and LeuRS is conserved through evolution and is believed to have been present when the tree of life split into three branches. In contrast, the editing site in class II Ala/ThrRS and ProRS is not absolutely conserved in the three kingdoms.

The class I subgroup comprising LeuRS, IleRS, and ValRS has particularly high levels of sequence and structural homology (Cusack et al. 2000). The three enzymes (hereafter, LIV-RS) are large monomeric proteins containing a long insertion called connective peptide 1 (CP1) corresponding to the editing domain. The fidelity of activation of the small nonpolar amino acids is still a real challenge for these enzymes, since these small substrates provide only a few chemical groups recognizable by the protein. Valine, which differs from isoleucine by a single methyl group, is activated by IleRS only 180-fold less efficiently than isoleucine (Eldred and Schimmel 1972). 
Threonine, an isostere of valine, is activated by ValRS at a rate of 1/250 of valine (Fersht and Kaethner 1976). LeuRS has been reported to misactivate multiple amino acids (Englisch et al. 1986; Apostol et al. 1997; Chen et al. 2000). Consequently, during evolution, LIV-RS enzymes have evolved pre- and post-transfer editing functions to increase the specificity of the aminoacylation reaction.

In the current study, we set out to understand the coevolution of the synthetic and editing sites in LeuRS, IleRS, and ValRS enzymes and find relics of their evolution in present-day synthetases. Because the editing domains of Aquifex aeolicus LeuRS (AaLeuRS), Escherichia coli IleRS (EcIleRS), and Bacillus stearothermophilus ValRS (BsValRS) can be isolated as active domains, they allowed examination of the editing activity independently of the synthetic activity (Lin et al. 1996; Zhao et al. 2005). We found that AaLeuRS and its isolated CP1 domain (AaLeu-CP1) edit the charged and mischarged noncognate $\mathrm{TRNA}^{\mathrm{Ile}}$ and $\mathrm{tRNA}^{\mathrm{Val}}$ in addition to Ile-tRNA ${ }^{\text {Leu }}$, as would be expected from a primitive LIVRS enzyme. A composite minihelix designed to carry the triple amino acid identity was charged by all three enzymes, but was only significantly hydrolyzed by the AaLeuRS isolated CP1 domain. These data indicate that AaLeuRS has retained or enlarged the hydrolytic function to edit the mischarged RNA issued from LeuRS, IleRS, and ValRS. Although this property can be considered as a functional relic, it can also result from a more recent adaptation to specific metabolic changes in the bacterium A. aeolicus. Together, these data support the hypothesis that LIV-RS enzymes have a common origin, and that the $A$. aeolicus $\mathrm{CP} 1$ has an intrinsic plasticity ready to evolve new editing specificities.

\section{RESULTS}

\section{Specificity of editing function of three class la aaRS}

Previous work showed that AaLeuRS, EcIleRS, BsValRS, and their corresponding isolated CP1 domains (AaLeu$\mathrm{CP} 1$, EcIle-CP1, and BsVal-CP1, respectively) carry posttransfer editing activity in vitro (Lin et al. 1996; Zhao et al. 2005). To gain insights into the specificity of their editing function, we constructed six substrates, including the three cognate aminoacylated tRNAs Leu-tRNA ${ }^{\text {Leu }}$, Ile-tRNA ${ }^{\text {Ile }}$, and Val-tRNA ${ }^{\mathrm{Val}}$, and the three misacylated tRNAs IletRNA $^{\text {Leu }}$, Val-tRNA ${ }^{\text {Ile }}$, and Thr-tRNA ${ }^{\text {Val }}$.

$B s$ ValRS hydrolyzed its usual mischarged product Thr$\mathrm{tRNA}^{\mathrm{Val}}$ rapidly. However, it was unable to hydrolyze the other five substrates, even at a high enzyme concentration $(5 \mu \mathrm{M})$. BsVal-CP1 was also specific for Thr-tRNA ${ }^{\mathrm{Val}}$ as previously shown (Fig. 1A,B; Lin et al. 1996).

Under the same conditions, EcIleRS efficiently hydrolyzed its natural mischarged substrate (Val-tRNA ${ }^{\text {Ile }}$ ). In addition, at a high enzyme concentration ( $5 \mu \mathrm{M})$, three noncognate tRNAs carrying the noncognate amino acids
Val-tRNA $^{\text {Val }}$, Thr-tRNA ${ }^{\text {Val }}$, and Leu-tRNA ${ }^{\text {Leu }}$ were hydrolyzed. However, at this high enzyme concentration, EcIleRS remained specific to its amino acid and IletRNA $^{\text {Leu }}$ was not hydrolyzed (Fig. 1C). EcIle-CP1 exhibited nearly the same pattern of editing, except that Leu-tRNA ${ }^{\text {Leu }}$ could not be hydrolyzed, and no preference for the naturally mischarged product Val-tRNA ${ }^{\text {Ile }}$ could be detected (Fig. 1D).

The last enzyme, AaLeuRS, showed the strongest editing activity. Using only $50 \mathrm{nM}$ AaLeuRS, hydrolysis of all aa-tRNAs, except Leu-tRNA ${ }^{\text {Leu }}$, could be detected (Fig. 1E, inset). The isolated $A a$ Leu-CP1 showed the same pattern of hydrolysis but with no preference for the recurrent mischarged product Ile-tRNA ${ }^{\text {Leu }}$ (Fig. 1F). However, the reaction was less efficient and required $5 \mu \mathrm{M}$ of isolated CP1 domain.

Next, we quantified the editing parameters of AaLeuRS for the different substrates (Table 1). The $k_{\text {cat }}$ app varied moderately from $1.45 \mathrm{sec}^{-1}$ for Ile-tRNA ${ }^{\text {Leu }}$ to $0.27 \mathrm{sec}^{-1}$ for Val-tRNA ${ }^{\mathrm{Val}}$, which is the poorest substrate. The Michaelian constant $K_{\mathrm{m}}$ was more severely affected and varied from $1.6 \mu \mathrm{M}$ to $25 \mu \mathrm{M}$. Ile-tRNA ${ }^{\text {Ile }}$ and Val-tRNA ${ }^{\text {Ile }}$ exhibited the lowest affinity and a loss of editing efficiency, with $k_{\text {cat }}{ }^{\text {app }}$ to $K_{\mathrm{m}}$ ratios of 21 and 20 for Ile-tRNA ${ }^{\text {Ile }}$ and Val-tRNA ${ }^{\text {Ile }}$, respectively, when compared with Ile-tRNA ${ }^{\text {Leu, }}$, the recurrent mischarged product of LeuRS.

\section{Design and aminoacylation of minihelix ${ }^{\mathrm{LIV}}$}

To mimic the possible ancient substrate of the primitive CP1 editing domain, a composite minihelix (hereafter, minihelix ${ }^{\text {LIV }}$ ) was designed by aligning the sequences of the acceptor stems and T $\psi \mathrm{C}$ loops of $A$. aeolicus $\mathrm{tRNA}^{\mathrm{Leu}}$ and E. coli tRNA ${ }^{\text {Leu }}$, $\mathrm{tRNA}^{\text {Ile }}$, and $\mathrm{tRNA}^{\text {Val }}$ (Fig. 2A). Invariant nucleotides and very conserved residues were chosen to constitute the minihelix ${ }^{\text {LIV }}$. For less conserved residues, we took into account the overrepresentation of tRNA $^{\text {Leu }}$ sequences and sometimes gave preference to the $\mathrm{tRNA}^{\text {Ile }}$ or $\mathrm{tRNA}^{\mathrm{Val}}$ nucleotide. The first $5^{\prime}$-terminal nucleotide was deleted to increase the flexibility of the single-stranded 3' end (Fig. 2B; Nordin and Schimmel 1999; Xu et al. 2004b). Leucine, isoleucine, valine, and threonine were attached to the minihelix ${ }^{\text {LIV }}$ by AaLeuRS, EcIleRS, EcValRS, and EcValRS-T ${ }_{222}$ P (Döring et al. 2001), respectively (Fig. 2C). AaLeuRS and EcIleRS efficiently aminoacylated minihelix ${ }^{\text {LIV }}$, compared with minihelix ${ }^{\text {Leu }}$ (Xu et al. 2004b) and minihelix ${ }^{\text {Ile }}$, respectively (Nordin and Schimmel 1999).

\section{AaLeu-CP1 hydrolyzes various species of aminoacyl-minihelix ${ }^{\text {LIV }}$}

The hydrolyses of the Leu-, Ile-, Val-, and Thr-minihelix ${ }^{\text {LIV }}$ by AaLeu-CP1, EcIle-CP1, BsVal-CP1, and Leu-CP1 from 
A

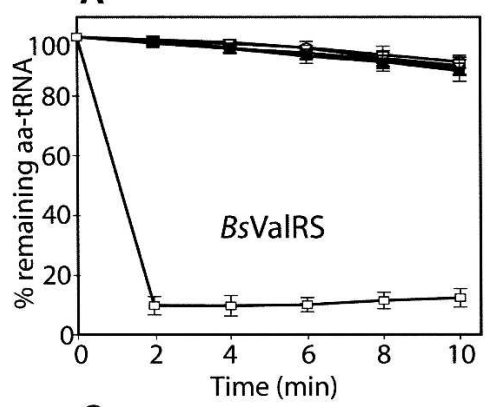

C

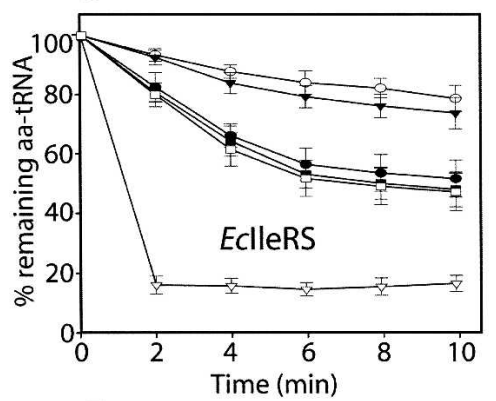

E

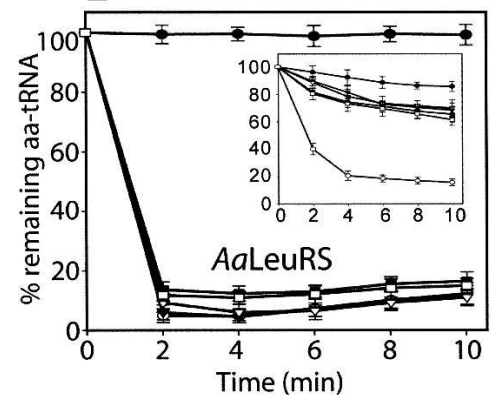

B

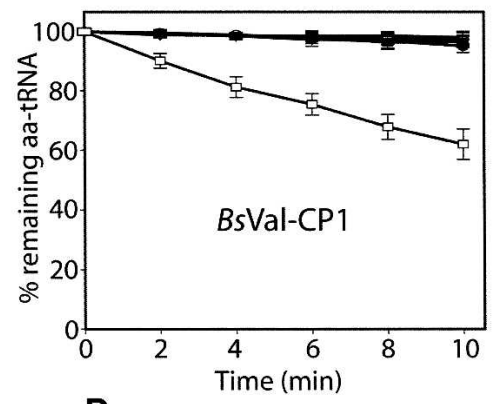

D
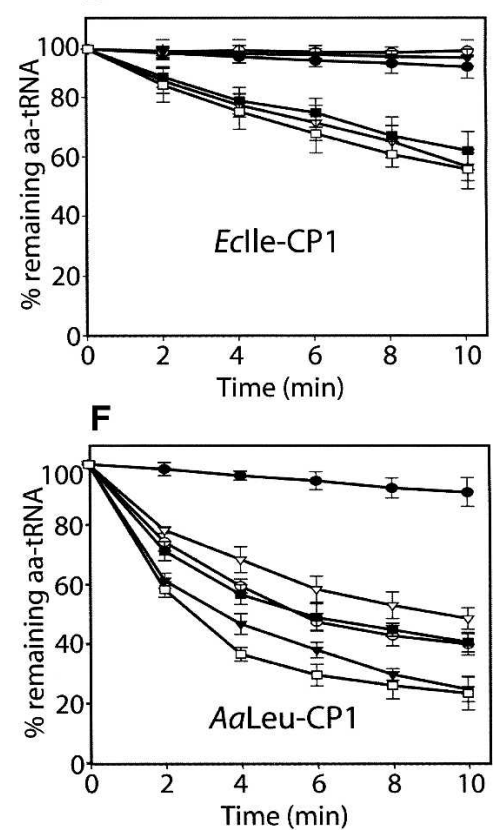

FIGURE 1. Editing of different aa-tRNAs by different aaRSs or editing domains. Deacylation of $\left[{ }^{3} \mathrm{H}\right]$ Leu-tRNA ${ }^{\text {Leu }}(\mathbf{O}),\left[{ }^{3} \mathrm{H}\right]$ Ile-tRNA ${ }^{\text {Leu }}(\mathrm{O}),\left[{ }^{3} \mathrm{H}\right]$ Ile-tRNA ${ }^{\text {Ile }}(\boldsymbol{\nabla})$, $\left[{ }^{3} \mathrm{H}\right]$ Val-tRNA ${ }^{\text {Ile }}(\nabla),\left[{ }^{3} \mathrm{H}\right]$ Val-tRNA ${ }^{\text {Val }}(\square)$, and $\left[{ }^{3} \mathrm{H}\right] \mathrm{Thr}^{-\mathrm{tRNA}}{ }^{\text {Val }}(\square)$ by $(A) 5 \mu \mathrm{M}$ BsValRS, (B) $5 \mu \mathrm{M}$ BsVal-CP1, (C) $5 \mu \mathrm{M}$ EcIleRS, (D) $5 \mu \mathrm{M}$ EcIle-CP1, (E) $5 \mu \mathrm{M}$ AaLeuRS ( $50 \mathrm{nM} A a$ LeuRS in the inset), and $(F) 5 \mu \mathrm{M}$ AaLeu-CP1. tRNAs originated from $(A-D)$ E. coli or $(E, F)$ A. aeolicus. The background hydrolysis of aminoacyl-tRNA in the absence of enzyme $(<15 \%$ over $10 \mathrm{~min}$ and $30 \%$ for LeutRNA $^{\text {Leu }}$ ) was subtracted from these plots for normalization purposes. Values were obtained from three independent determinations.

E. coli (EcLeu-CP1) were assayed. Here we added EcLeuCP1 because it can hydrolyze a mischarged minihelix ${ }^{\text {Leu }}$ and serve as a control for the leucine-aminoacylating system (Zhao et al. 2005). The different CP1 domains were tested at the same concentration of $5 \mu \mathrm{M}$. AaLeu-CP1 hydrolyzed all substrates except the "cognate product" Leu-minihelix ${ }^{\text {LIV }}$ (Fig. 3). EcLeu-CP1 specifically hydrolyzed only the Ile-minihelix ${ }^{\mathrm{LIV}}$, demonstrating that the AaLeu-CP1 has ambiguous substrate recognition. The two other isolated CP1 domains, EcIle-CP1 and BsVal$\mathrm{CP} 1$, did not hydrolyze any of the four substrates (Fig. 3). Positive controls using Val-tRNA ${ }^{\text {Ile }}$ and Thr-tRNA ${ }^{\text {Val }}$ for EcIle-CP1 and BsVal-CP1, respectively, were also carried out (data not shown).

\section{DISCUSSION}

The modular architecture of present-day
aaRS mirrors their step-by-step evolution

AaRS form a family of enzymes that have been intensely studied. These modular enzymes are thought to be derived from two ancestors that used two distinct architectures to construct their catalytic sites. These catalytic domains represent the ancient, historical enzyme that carries determinants for activation of the amino acid and for binding to the acceptor minihelix of tRNA (Schimmel and Ribas de Pouplana 1995). Indeed, helical substrates that mimic the acceptor stem of tRNA are aminoacylated by many present-day tRNA synthetases (Schimmel and Ribas de Pouplana 2001), suggesting that primordial tRNAs might have been minihelices. During evolution, the ancient minihelix might have been duplicated to give rise to the present tRNAs with four major arms (Rodin et al. 1996). At the same time, additional motifs and domains were added to the two core structures of the primitive synthetases. These additions facilitated interactions with the new parts of the tRNA distal to the acceptor end. With the increasing number of amino acids incorporated in the genetic code, these enzymes were exposed to recognition and specificity problems. Additional domains, called editing domains, were added to improve the precision of the genetic code and clear mischarged tRNAs. In this way, the precision of the genetic code was continuously improved during the long process of evolution. Nowadays, the precision of the modern genetic code is still sharply dependent on the editing function of tRNA synthetases (Döring et al. 2001). For that reason, the appearance of editing activity in some tRNA synthetases could have arisen very early, before their radiation into 20 different enzymes (Schimmel and Ribas de Pouplana 2001). For instance, the CP1 editing domain found in LIV-RS enzymes is found in all branches of the three kingdoms, even the deepest, suggesting that it was added before the radiation of the enzymes from the class Ia common ancestor.

\section{Does $A$. aeolicus LeuRS carry functional relics or develop new properties?}

We previously reported that LeuRS from the deep-branching bacterium A. aeolicus carries basic features supposed to have been present in ancient aaRS. First, it has a unique $\alpha \beta$-heterodimeric structure with separated catalytic and 
TABLE 1. Apparent editing parameters of AaLeuRS for different aa-tRNAs

\begin{tabular}{|c|c|c|c|c|c|}
\hline Substrate & $k_{\text {cat }}^{\text {app }}\left(\mathrm{sec}^{-1}\right)$ & $K_{\mathrm{m}}^{\text {app }}(\mu \mathrm{M})$ & $\begin{array}{l}k_{\text {cat }}{ }^{\mathrm{app}} / K_{\mathrm{m}}{ }^{\mathrm{app}} \\
\left(\mu \mathrm{M}^{-1} \mathrm{sec}^{-1}\right)\end{array}$ & $\begin{array}{c}k_{\text {cat }}{ }^{\text {app }} / K_{\mathrm{m}}{ }^{\text {app }} \\
\text { (relative) }\end{array}$ & $\begin{array}{c}\text { Loss of } \\
\text { efficiency (-fold) }\end{array}$ \\
\hline$\left[{ }^{3} \mathrm{H}\right] \| l e-t R N A^{\text {Leu }}$ & $1.45 \pm 0.2$ & $1.6 \pm 0.2$ & 0.9 & 1 & 1 \\
\hline$\left[{ }^{3} \mathrm{H}\right]$ Ile-tRNA ${ }^{\text {Ile }}$ & $1.08 \pm 0.2$ & $25 \pm 4$ & 0.04 & 0.05 & 21 \\
\hline$\left[{ }^{3} \mathrm{H}\right]$ Val-tRNA ${ }^{l l e}$ & $0.49 \pm 0.1$ & $11 \pm 2$ & 0.04 & 0.05 & 20 \\
\hline$\left[{ }^{3} \mathrm{H}\right]$ Val-tRNA ${ }^{\text {Val }}$ & $0.27 \pm 0.1$ & $4.5 \pm 0.7$ & 0.06 & 0.07 & 15 \\
\hline$\left[{ }^{3} \mathrm{H}\right]$ Thr-tRNA ${ }^{\mathrm{Val}}$ & $0.31 \pm 0.1$ & $5.0 \pm 0.6$ & 0.06 & 0.07 & 15 \\
\hline
\end{tabular}

Data are the average of three independent determinations. $k_{\mathrm{cat}}{ }^{\text {app }}$ and $K_{\mathrm{m}}{ }^{\text {app }}$ are apparent $k_{\mathrm{cat}}$ and $K_{\mathrm{m}}$, respectively. For clarity, the values of the three last columns were rounded off.

tRNA-binding sites (Xu et al. 2002). Such an organization supports the tRNA/synthetase coevolution theory that predicts sequential addition of tRNA and synthetase domains (see above). Second, the AaLeuRS editing domain expressed as a freestanding domain is able to edit mischarged tRNA $^{\text {Leu }}$ and minihelix ${ }^{\text {Leu, }}$, thanks to a crucial specific 20-amino acid peptide inserted in this editing domain (Zhao et al. 2005). Third, we showed that the fusion of the tRNA-binding $\beta$ subunit of $A a$ LeuRS with the E. coli editing domain could activate the editing function (Zhao et al. 2005). Altogether, these properties suggest that AaLeuRS has a remarkable capacity to transfer autonomous active modules, which is consistent with the idea that modern synthetases arose after exchange of small idiosyncratic domains.

Nevertheless, the exact origin of the remarkable properties discovered in the $A a$ LeuRS remains yet unsolved. According to the deep rooting of the Aquifex genus in the tree of life, we previously proposed that these features are ancestral features of LeuRS (Zhao et al. 2005). In the present work we describe another unusual functional property found in the AaLeuRS. Although it might fit in the ancestral model, it might also reflect recent evolution of the A. aeolicus LeuRS in response to specific metabolic changes.

\section{Editing of $A$. aeolicus LeuRS is highly ambiguous}

LIV-RS enzymes display remarkable similarities at both sequence and structural levels, which can be considered as relics from their common lineage. Schematic trees of evolution of LeuRS, IleRS, and ValRS were drawn according to sequence-based alignments (Fig. 4; Brown and Doolittle 1995). In this study, we add functional evidence to support their common origin. As a model, we used A. aeolicus AaLeuRS, an enzyme that contains remarkable functional relics from synthetase evolution (see above). We show here that AaLeuRS recognizes the noncognate tRNA ${ }^{\text {Ile }}$ and $\mathrm{tRNA}^{\mathrm{Val}}$ in addition to $\mathrm{RNA}^{\mathrm{Leu}}$ in the editing reaction, as it would have done by a primordial LeuRS just derived from the precursor of the LIV-RS enzyme. A total of five noncognate substrates were hydrolyzed, whereas the cognate product of LeuRS, Leu-tRNA ${ }^{\text {Leu }}$, was unaffected. However, a clear preference for the cognate mischarged
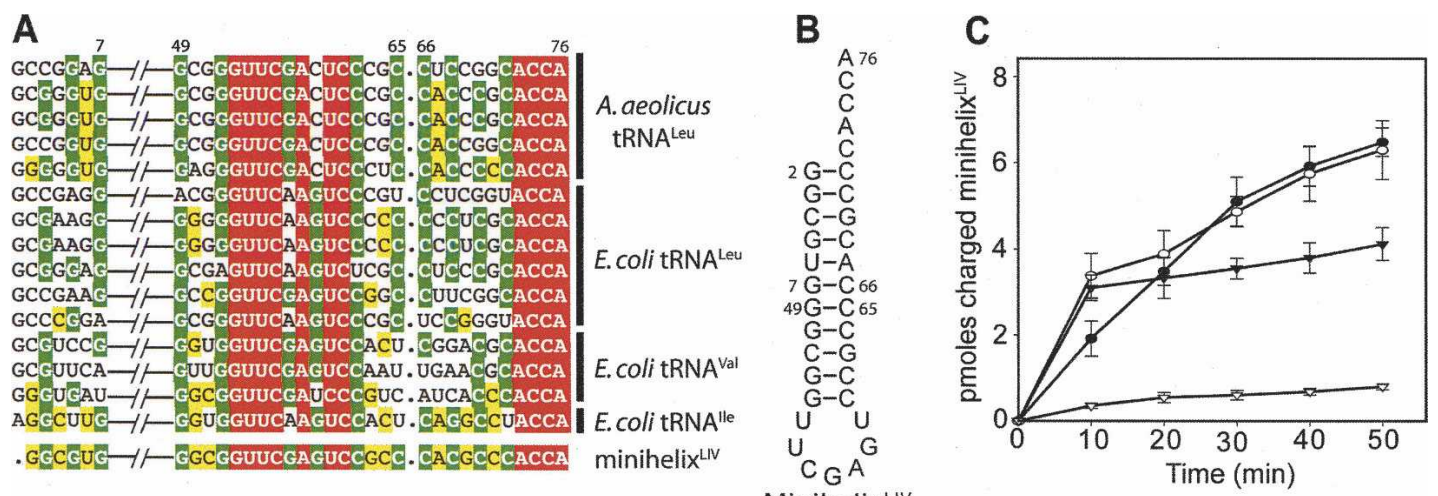

FIGURE 2. Sequence and aminoacylation of minihelix ${ }^{\mathrm{LIV}}$. (A) Partial alignment from A. aeolicus tRNAs ${ }^{\mathrm{Leu}}$ and E. coli tRNAs $^{\mathrm{Leu}}$, tRNAs ${ }^{\mathrm{Val}}$, and tRNA $^{\text {Ile }}$. The minihelix ${ }^{\text {LIV }}$ sequence was designed based on sequence conservation. Some nucleotides (highlighted in yellow) were chosen by taking into account the over-representation of tRNA ${ }^{\text {Leu }}$ sequences. Invariant bases are highlighted in red, very conserved bases in green. $(B)$ Secondary structure of minihelix ${ }^{\mathrm{LIV}}$. (C) Attachment of leucine, isoleucine, valine, and threonine to minihelix ${ }^{\mathrm{LIV}}$ by $A a \operatorname{LeuRS}(\mathbf{O})$, EcIleRS (O), EcValRS $(\nabla)$, and $\mathrm{T}_{222} \mathrm{P} E c$ ValRS $(\nabla)$ under the same conditions. Reactions were assayed at $25^{\circ} \mathrm{C}$ with $25 \mu \mathrm{M}$ minihelix ${ }^{\mathrm{LIV}}$ and $5 \mu \mathrm{M}$ enzyme, respectively. No RNA controls were used to correct for background rates ( $<5 \%$ of the minihelix ${ }^{\text {LIV }}$ charging). 
A

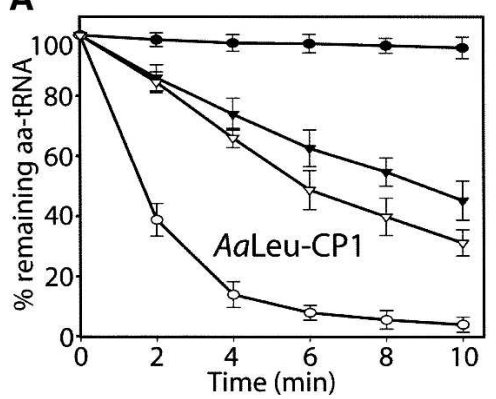

C

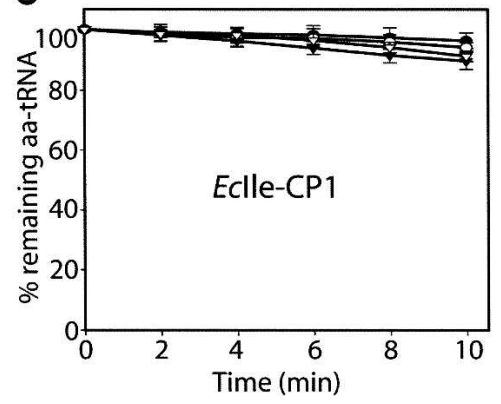

B

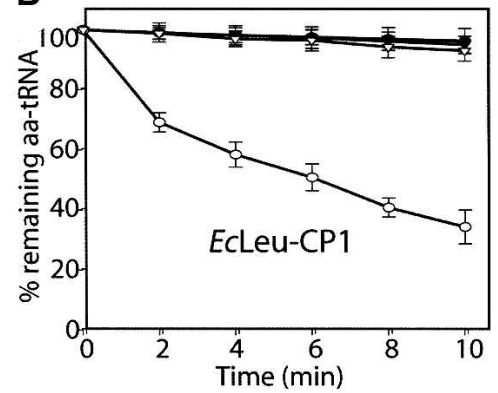

D

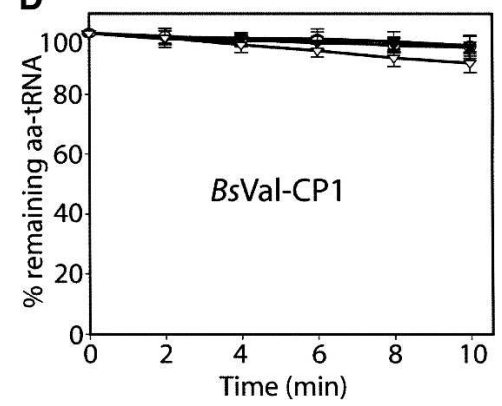

FIGURE 3. Editing of different aa-minihelices ${ }^{\text {LIV }}$ by various editing domains. Deacylation of $\left[{ }^{3} \mathrm{H}\right]$ Leu-minihelix ${ }^{\text {LIV }}(\bullet),\left[{ }^{3} \mathrm{H}\right]$ Ile-minihelix ${ }^{\text {LIV }}(\bigcirc),\left[{ }^{3} \mathrm{H}\right]$ Val$\operatorname{minihelix}^{\mathrm{LIV}}(\boldsymbol{\nabla})$, and $\left[{ }^{3} \mathrm{H}\right] \mathrm{Thr}-$ minihelix $^{\mathrm{LIV}}(\nabla)$ by $(A) 5 \mu \mathrm{M}$ AaLeu-CP1, $(B) 5 \mu \mathrm{M}$ EcLeu-CP1, (C) $5 \mu \mathrm{M}$ EcIle-CP1, and (D) $5 \mu \mathrm{M}$ BsVal-CP1.

substrate (Ile-tRNA ${ }^{\text {Leu }}$ ) was observed when compared to the noncognate aminoacyl-tRNA ${ }^{\text {Ile }}$ and tRNA ${ }^{\text {Val }}$. This suggests that the hydrolytic function is not completely relaxed but follows some regulations.

The enlarged editing capacity here characterized might be considered as a functional relic that predates the split of the LIV-RS precursor in the three modern aaRS. In the ancient time, editing might have been essential to clear other compounds that differed from leucine, isoleucine, and valine. Small metabolites or nonproteinous amino acids might have been targeted by the editing function. After radiation from the progenitor, the three new class Ia enzymes (LeuRS, IleRS, and ValRS) would have to focus their editing function to discriminate between the neighboring class Ia amino acids. For an unexplained reason, the editing function of AaLeuRS has been maintained in a relatively ambiguous state compared to IleRS and ValRS.

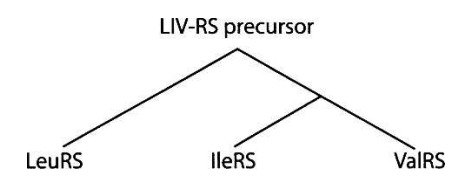

FIGURE 4. Schematic trees of evolution of class Ia LeuRS, IleRS, and ValRS. Schematic tree of evolution of class Ia LeuRS, IleRS, and ValRS drawn according to sequence-based alignments (Brown and Doolittle 1995). The phylogeny shows that IleRS and ValRS group closely together to the exclusion of LeuRS, which forms an outgroup.
An alternative explanation that is equally well supported by the data might be proposed. AaLeuRS's expanded editing ability might be a recent adaptation to the high temperature and extreme growth conditions encountered by the organism. A. aeolicus is an obligate chemolithoautotroph and the most hyperthermophilic bacterium known. Under this metabolic constraint, AaLeuRS might have acquired an expanded editing ability in response to the specific compounds generated in these extreme conditions.

\section{AaLeu-CP1 acts as a freestanding editing domain}

In this study, we show that $A a$ Leu-CP1 is able to recognize and hydrolyze the Ile-, Thr-, and Valminihelix ${ }^{\mathrm{LIV}}$, with a marked preference for Ileminihelix ${ }^{\text {LIV }}$. This further highlights the archaic properties of AaLeuRS and its CP1 editing domain that is active as a freestanding domain. Recent studies showed that freestanding proofreading domains still exist in the present-day protein synthesis machinery. The $\mathrm{YbaK}$ and ProX proteins, which are homologous to the class II ProRS editing domain, are freestanding editing domains that edit Cys-tRNA ${ }^{\text {Pro }}$ and Ala-tRNA ${ }^{\text {Pro }}$, respectively (An and Musier-Forsyth 2004; Ruan and Söll 2005). AlaX protein is a freestanding homolog from the AlaRS editing domain (Ahel et al. 2003) that hydrolyzes Ser-tRNA ${ }^{\text {Ala }}$ and Gly-tRNA ${ }^{\text {Ala }}$. ThrRS-ed is a freestanding editing domain found in Archaea that hydrolyzes SertRNA $^{\text {Thr }}$ (Korencic et al. 2004). Although these deacylases are sometimes dispensable for cell growth under standard growth conditions, they play a significant role under extreme cell growth conditions that favor tRNA mischarging (Korencic et al. 2004). Our results show that the CP1 domain from AaLeuRS preferentially edits the mischarged Ile-tRNA ${ }^{\text {Leu }}$ but can also edit several similar aa-tRNAs. The lack of specificity is increased when the editing domain is expressed as a freestanding domain. These characteristics might provide A. aeolicus with two clear advantages. First, the multisubstrate specificity of CP1 suggests that it has preserved its ability to evolve the editing function, a property that would favor adaptation of the bacterium to new mischarged substrates. Second, under some physiological conditions, one can imagine that a significant number of $\alpha$ subunits of $A$. aeolicus LeuRS might be released in the cell and act as freestanding CP1s. These domains would probably have an enlarged set of mischarged tRNAs to deacylate. For the cell, this would be an easy and economical way to express two products with different properties starting from a single gene.

To conclude, the expanded editing properties of AaLeuRS might be a real ancestral editing activity resulting 
from the slow-evolving rate of the Aquifex genus or it might be a recent adaptation to specific Aquifex metabolic constraints. More investigations will require purification and characterization of the editing activities of all LIV-RS enzymes from $A$. aeolicus in addition to those from close primitive bacteria (Thermotoga, Thermus) and primitive eukaryotes (Diplomonads) and archaea (Korarchaeota) in order to check the distribution of the expanded editing ability in deeply rooted organisms.

\section{MATERIALS AND METHODS}

\section{Materials}

L-Leucine, L-isoleucine, L-valine, L-threonine, 5'-GMP, ATP, GTP, CTP, UTP, tetrasodium pyrophosphate, inorganic pyrophosphatase, and DTT were purchased from Sigma. L- $\left[{ }^{3} \mathrm{H}\right]$ Leucine, $\mathrm{L}-\left[{ }^{3} \mathrm{H}\right]$ isoleucine, $\mathrm{L}-\left[{ }^{3} \mathrm{H}\right]$ valine, and $\mathrm{L}-\left[{ }^{3} \mathrm{H}\right]$ threonine $(1 \mathrm{mCi} / \mathrm{mL})$ were obtained from Amersham Life Sciences. Kinase, ligase, RNasin (ribonuclease inhibitor), isopropyl $\beta$-D-thiogalactoside (IPTG), and all restriction endonucleases were obtained from the Sangon Company. T7 RNA polymerase was purified as described (Li et al. 1999). Plasmids pET28a and pET30a were purchased from Novagen (Biosciences Inc.). The bacterial BL21-Codon Plus (DE3)-RIL strain was purchased from Stratagene. Nickelnitrilotriacetic (Ni-NTA) Superflow resin was obtained from QIAGEN Inc. GF/C and DE-81 filters were obtained from the Whatman Company.

\section{Preparation of tRNAs and proteins}

DNA fragments encoding CP1 domains and enzymes were amplified by PCR. Mutated synthetases were generated by a two-step PCR method. PCR products were cloned into either pET28a or pET30a. CP1 domains were designed as previously described (Lin et al. 1996; Zhao et al. 2005). A. aeolicus LeuRS (AaLeuRS), E. coli IleRS (EcIleRS), B. stearothermophilus ValRS (BsValRS), and the corresponding CP1 domains were overexpressed in E. coli and purified (Lin et al. 1996; Zhao et al. 2005). A. aeolicus and E. coli tRNA $^{\text {Ile }}$ (GAU) were overexpressed in E. coli and purified as previously described (Xu et al. 2002). Transcripts from A. aeolicus and E. coli tRNA ${ }^{\text {Leu }}$ (GAG) and tRNA ${ }^{\text {Val }}$ (UAC) were prepared according to $\mathrm{Xu}$ et al. (2004b).

\section{Design and preparation of minihelix ${ }^{\text {LIV }}$}

The sequence of the minihelix ${ }^{\mathrm{LIV}}$ was designed according to the acceptor stems and T $\psi C$ loops of $A$. aeolicus tRNA ${ }^{\text {Leu }}$ (GAG), and tRNA $^{\text {Leu }}(\mathrm{GAG}), \mathrm{tRNA}^{\text {Ile }}(\mathrm{GAU})$, and $\mathrm{tRNA}^{\mathrm{Val}}$ (UAC) from E. coli. RNA from minihelix ${ }^{\text {LIV }}$ was obtained by T7 RNA transcription of annealed oligodeoxynucleotides. In vitro transcription and purification of minihelix ${ }^{\text {LIV }}$ followed the protocol previously described (Xu et al. 2004b).

\section{Aminoacyl-RNA substrate preparation}

A. aeolicus tRNA ${ }^{\text {Leu }}$ was charged with $\left[{ }^{3} \mathrm{H}\right]$ Leu by AaLeuRS to give $\left[{ }^{3} \mathrm{H}\right]$ Leu-tRNA ${ }^{\mathrm{Leu}}$. E. coli tRNA $^{\text {Ile }}$ and E. coli $\mathrm{tRNA}^{\mathrm{Val}}$ were charged with $\left[{ }^{3} \mathrm{H}\right]$ Ile by EcIleRS and with $\left[{ }^{3} \mathrm{H}\right]$ Val by EcValRS, respectively. Mischarged products $\left[{ }^{3} \mathrm{H}\right]$ Ile-tRNA ${ }^{\text {Leu }},\left[{ }^{3} \mathrm{H}\right]$ ValtRNA ${ }^{\text {Ile }}$, and $\left[{ }^{3} \mathrm{H}\right]$ Thr-tRNA ${ }^{\text {Val }}$ were prepared using the editingdeficient mutants EcLeuRS-T ${ }_{252} \mathrm{E}$ (Xu et al. 2004a), EcIleRS- ${ }_{242} \mathrm{P}$ (Hendrickson et al. 2000), and EcValRS- $\mathrm{T}_{222} \mathrm{P}$ (Döring et al. 2001), respectively. Aminoacylation of minihelix ${ }^{\text {LIV }}$ was carried out under the same conditions as minihelix ${ }^{\text {Ile }}$ (Nordin and Schimmel 1999). Two sets of mischarged tRNAs originating from E. coli or A. aeolicus were tested. No significant differences were detected in their editing rates regardless of their origins.

\section{Hydrolytic capacity assays}

The hydrolytic activities of different isolated CP1 domains and enzymes toward the different $\left[{ }^{3} \mathrm{H}\right]$ aa-tRNA were measured in reactions containing $100 \mathrm{mM}$ Tris- $\mathrm{HCl}(\mathrm{pH} 7.5), 30 \mathrm{mM} \mathrm{KCl}$, $12 \mathrm{mM} \mathrm{MgCl}_{2}, 0.5 \mathrm{mM} \mathrm{DTT}$, and $1 \mu \mathrm{M}\left[{ }^{3} \mathrm{H}\right]$ aa-tRNA at $37^{\circ} \mathrm{C}$. Reactions were initiated by adding isolated CP1 domain $(5 \mu \mathrm{M})$ or enzyme $(50 \mathrm{nM}$ or $5 \mu \mathrm{M})$. For minihelix ${ }^{\mathrm{LIV}}$, the editing activities were measured at $25^{\circ} \mathrm{C}$ in the presence of $1 \mu \mathrm{M}\left[{ }^{3} \mathrm{H}\right]$-aaminihelix $^{\mathrm{LIV}}$ and $5 \mu \mathrm{M}$ the different CP1 domains. At various time intervals, aliquots were quenched and precipitated with 5\% trichloroacetic acid (Zhao et al. 2005). The editing parameters $\left(K_{\mathrm{m}}{ }^{\text {app }}\right.$ and $k_{\text {cat }}{ }^{\text {app }}$ ) of $A a$ LeuRS for the different aminoacyl-tRNAs were measured using $100 \mathrm{nM}$ AaLeuRS. Aminoacyl-tRNA concentrations ranged from 0.5 to $40 \mu \mathrm{M}$.

\section{ACKNOWLEDGMENTS}

We thank T. Ueda and B.A. Roe for the E. coli ValRS gene and B. stearothermophilus strain, respectively. We are grateful to the anonymous referee who suggested an alternative interpretation of our results. This work was funded by the Natural Science Foundation of China (Grants 30330180 and 30570380), 973 project in China (Grant 2005CB724600), and the exchange program between CAS and CNRS (CNRS/DREI \#18141).

Received July 13, 2006; accepted October 2, 2006.

\section{REFERENCES}

Ahel, I., Korencic, D., Ibba, M., and Söll, D. 2003. Trans-editing of mischarged tRNAs. Proc. Natl. Acad. Sci. 100: 15422-15427.

An, S. and Musier-Forsyth, K. 2004. Trans-editing of Cys-tRNA ${ }^{\text {Pro }}$ by Haemophilus influenzae YbaK protein. J. Biol. Chem. 279: 42359-42362.

Apostol, I., Levine, J., Lippincott, J., Leach, J., Hess, E., Glascock, C., Weickert, M., and Blackmore, R. 1997. Incorporation of norvaline at leucine positions in recombinant human hemoglobin expressed in Escherichia coli. J. Biol. Chem. 272: 28980-28988.

Beebe, K., Ribas de Pouplana, L., and Schimmel, P. 2003. Elucidation of tRNA-dependent editing by a class II tRNA synthetase and significance for cell viability. EMBO J. 22: 668-675.

Beuning, P.J. and Musier-Forsyth, K. 2000. Hydrolytic editing by a class II aminoacyl-tRNA synthetase. Proc. Natl. Acad. Sci. 97: 8916-8920.

Brown, J. and Doolittle, W. 1995. Root of the universal tree of life based on ancient aminoacyl-tRNA synthetase gene duplications. Proc. Natl. Acad. Sci. 92: 2441-2445.

Chen, J.F., Guo, N.N., Li, T., Wang, E.D., and Wang, Y.L. 2000. CP1 domain in Escherichia coli leucyl-tRNA synthetase is crucial for its editing function. Biochemistry 39: 6726-6731.

Cusack, S., Yaremchuk, A., and Tukalo, M. 2000. The $2 \AA$ crystal structure of leucyl-tRNA synthetase and its complex with a leucyladenylate analogue. EMBO J. 19: 2351-2361. 
Dock-Bregeon, A., Sankaranarayanan, R., Romby, P., Caillet, J., Springer, M., Rees, B., Francklyn, C.S., Ehresmann, C., and Moras, D. 2000. Transfer RNA-mediated editing in threonyl-tRNA synthetase. The class II solution to the double discrimination problem. Cell 103: 877-884.

Döring, V., Mootz, H.D., Nangle, L.A., Hendrickson, T.L., de CrécyLagard, V., Schimmel, P., and Marlière, P. 2001. Enlarging the amino acid set of Escherichia coli by infiltration of the valine coding pathway. Science 292: 501-504.

Eldred, E.W. and Schimmel, P. 1972. Rapid deacylation by isoleucyl transfer ribonucleic acid synthetase of isoleucine-specific transfer ribonucleic acid aminoacylated with valine. J. Biol. Chem. 247: 2961-2964.

Englisch, S., Englisch, U., von der Haar, F., and Cramer, F. 1986. The proofreading of hydroxy analogues of leucine and isoleucine by leucyl-tRNA synthetases from E. coli and yeast. Nucleic Acids Res. 14: 7529-7539.

Eriani, G., Delarue, M., Poch, O., Gangloff, J., and Moras, D. 1990. Partition of tRNA synthetases into two classes based on mutually exclusive sets of sequence motifs. Nature 347: 203-206.

Fersht, A. and Kaethner, M. 1976. Enzyme hyperspecificity. Rejection of threonine by the valyl-tRNA synthetase by misacylation and hydrolytic editing. Biochemistry 15: 3342-3346.

Hendrickson, T.L., Nomanbhoy, T.K., and Schimmel, P. 2000. Errors from selective disruption of the editing center in a tRNA synthetase. Biochemistry 39: 8180-8186.

Ibba, M. and Söll, D. 2000. Aminoacyl-tRNA synthesis. Annu. Rev. Biochem. 69: 617-650.

Ishijima, J., Uchida, Y., Kuroishi, C., Tuzuki, C., Takahashi, N., Okazaki, N., Yutani, K., and Miyano, M. 2006. Crystal structure of alanyl-tRNA synthetase editing-domain homolog (PH0574) from a hyperthermophile, Pyrococcus horikoshii OT3 at $1.45 \AA$ resolution. Proteins 62: 1133-1137.

Korencic, D., Ahel, I., Schelert, J., Sacher, M., Ruan, B., Stathopoulos, C., Blum, P., Ibba, M., and Söll, D. 2004. A freestanding proofreading domain is required for protein synthesis quality control in Archaea. Proc. Natl. Acad. Sci. 101: 1026010265.

Kotik-Kogan, O., Moor, N., Tworowski, D., and Safro, M. 2005. Structural basis for discrimination of L-phenylalanine from L-tyrosine by phenylalanyl-tRNA synthetase. Structure 13: 17991807.

Li, Y., Wang, E., and Wang, Y. 1999. A modified procedure for fast purification of T7 RNA polymerase. Protein Expr. Purif. 16: 355-358.
Lin, L., Hale, S.P., and Schimmel, P. 1996. Aminoacylation error correction. Nature 384: 33-34.

Nordin, B. and Schimmel, P. 1999. RNA determinants for translational editing. Mischarging a minihelix substrate by a tRNA synthetase. J. Biol. Chem. 274: 6835-6838.

Nureki, O., Vassylyev, D., Tateno, M., Shimada, A., Nakama, T., Fukai, S., Konno, M., Hendrickson, T., Schimmel, P., and Yokoyama, S. 1998. Enzyme structure with two catalytic sites for double-sieve selection of substrate. Science 280: 578-582.

Rodin, S., Rodin, A., and Ohno, S. 1996. The presence of codonanticodon pairs in the acceptor stem of tRNAs. Proc. Natl. Acad. Sci. 93: 4537-4542.

Roy, H., Ling, J., Irnov, M., and Ibba, M. 2005. Post-transfer editing in vitro and in vivo by the $\beta$ subunit of phenylalanyl-tRNA synthetase. EMBO J. 23: 4639-4648.

Ruan, B. and Söll, D. 2005. The bacterial YbaK protein is a CystRNA $^{\text {Pro }}$ and Cys-tRNA ${ }^{\text {Cys }}$ deacylase. J. Biol. Chem. 280: 2588725891.

Schimmel, P. 1987. Aminoacyl-tRNA synthetases: General scheme of structure-function relationships in the polypeptides and recognition of transfer RNAs. Annu. Rev. Biochem. 56: 125-158.

Schimmel, P. and Ribas de Pouplana, L. 1995. Transfer RNA: From minihelix to genetic code. Cell 81: 983-986.

Schimmel, P. and Ribas de Pouplana, L. 2001. Formation of two classes of tRNA synthetases in relation to editing functions and genetic code. Cold Spring Harb. Symp. Quant. Biol. 66: 161-166.

Wong, F., Beuning, P., Silvers, C., and Musier-Forsyth, K. 2003. An isolated class II aminoacyl-tRNA synthetase insertion domain is functional in amino acid editing. J. Biol. Chem. 278: 52857-52864.

Xu, M.G., Chen, J.F., Martin, F., Zhao, M.W., Eriani, G., and Wang, E.D. 2002. Leucyl-tRNA synthetase consisting of two subunits from hyperthermophilic bacteria Aquifex aeolicus. J. Biol. Chem. 277: 41590-41596.

Xu, M.G., Li, J., Du, X., and Wang, E.D. 2004a. Groups on the side chain of T252 in Escherichia coli leucyl-tRNA synthetase are important for discrimination of amino acids and cell viability. Biochem. Biophys. Res. Comm. 318: 11-16.

Xu, M.G., Zhao, M.W., and Wang, E.D. 2004b. Leucyl-tRNA synthetase from the hyperthermophilic bacterium Aquifex aeolicus recognizes minihelices. J. Biol. Chem. 279: 32151-32158.

Zhao, M.W., Zhu, B., Hao, R., Xu, M.G., Eriani, G., and Wang, E.D. 2005. Leucyl-tRNA synthetase from the ancestral bacterium Aquifex aeolicus contains relics of synthetase evolution. EMBO J. 24: $1430-1439$. 

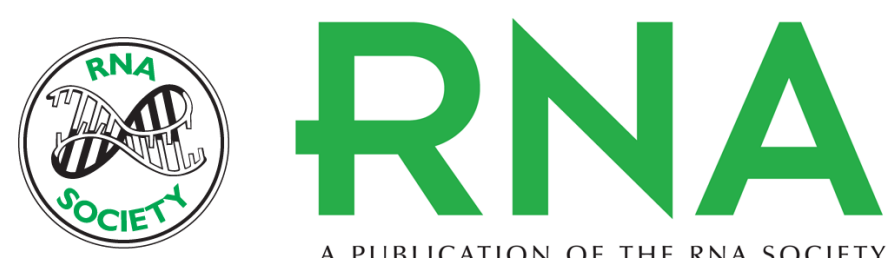

A PUBLICATION OF THE RNA SOCIETY

\section{A present-day aminoacyl-tRNA synthetase with ancestral editing properties}

Bin Zhu, Ming-Wei Zhao, Gilbert Eriani, et al.

RNA 2007 13: 15-21 originally published online November 9, 2006

Access the most recent version at doi:10.1261/rna.228707

References This article cites 33 articles, 18 of which can be accessed free at:

http://rnajournal.cshlp.org/content/13/1/15.full.html\#ref-list-1

License

Email Alerting Receive free email alerts when new articles cite this article - sign up in the box at the Service top right corner of the article or click here.

To subscribe to RNA go to:

http://rnajournal.cshlp.org/subscriptions 